\title{
A COMPARISON OF TWO APPROACHES TO PARAINCONSISTENCY: Flemish and Polish
}

\begin{abstract}
In this paper we present a comparison of certain inconsistency adaptive logics and Jaśkowski's logic.
\end{abstract}

\section{Introduction}

One of the first formal ways of handling inconsistency was presented in [11]. The approach opened the way to further investigations concerning paraconsistent logic. In time the importance of so called $M$-fragments of a given modal logic was also discovered.

Another approach is given by D. Batens. The whole project started with the paper [1]. The idea is very attractive and can be applied to many different logical calculi.

\section{Inconsistency adaptive logics}

The adaptive logics are built with the help of two consequences relations: a weaker one (in the case of inconsistency adaptive logics it is a paraconsistent logic) and a stronger (classical logic). You can always derive a conclusion from given premises using the first kind of consequences relation, while in some cases we can use the second one. The cases in which the stronger consequence relation is allowed are determined by a given strategy. Logics 
ACLuN1 and ACLuN2 are the most famous. In what follows, we will refer only to the propositional part of these logics, since the most important features of the predicative formulations are retained in the propositional versions.

In both these logics, the logic $\mathbf{C L u N}$ is the lower limit logic. The propositional case of the logic CLuN is defined as the full positive classical logic plus the law of excluded middle.

Let us recall a very important theorem from [2] which was originally expressed for the propositional case.

$\vdash_{\mathbf{C L}} A$ iff there are $C_{1}, \ldots, C_{n}(n \geqslant 0)$ that $\vdash_{\mathbf{C L u N}} \operatorname{DEK}\left(C_{1}, \ldots, C_{n}\right) \vee A$.

This suggests rules of inference for inconsistency adaptive logics.

In the proofs of inconsistency adaptive logics every formula is added under assumption of consistent behavior of some set of formulas. If the set of assumptions under which a given formula is added to the proof, is the empty set, we say that the formula appears unconditionally in the proof.

We only recall the meta-rules which govern the inferences for ACLuN1 logic. It is enough for our purposes since in the case of consistent sets of premisses both consequences coincide, while in general if $X \vdash_{\text {ACLuN1 }} A$ then $X \vdash_{\text {ACLuN2 }} A$. For the exhaustive formulations of semantics and syntax of both logics, see for example [3].

We say that a formula of the form $\left(C_{1} \wedge \sim C_{1}\right) \vee \cdots \vee\left(C_{m} \wedge \sim C_{m}\right)$ is a minimally inconsistent disjunction in a given proof if it appears in the proof unconditionally and no formula of the form $\left(C_{i_{1}} \wedge \sim C_{i_{1}}\right) \vee \cdots \vee\left(C_{i_{k}} \wedge \sim C_{i_{k}}\right)$ where $k<m$, appears in the given proof unconditionally.

A formula of the form $\left(C_{1} \wedge \sim C_{1}\right) \vee \cdots \vee\left(C_{m} \wedge \sim C_{m}\right)$ is a minimally inconsistent consequence of a given set $X$ iff it is a consequence of $X$ in the sense of the logic CLuN and no formula of the form $\left(C_{i_{1}} \wedge \sim C_{i_{1}}\right) \vee \cdots \vee$ $\left(C_{i_{k}} \wedge \sim C_{i_{k}}\right)$ where $k<m$, is a consequence of $X$. Here are the rules:

RU $\quad$ If $\vdash_{\text {CLuN }}\left(A_{1} \wedge \cdots \wedge A_{n}\right) \rightarrow B$ and $A_{1}, \ldots, A_{n}$ appears in the proof, $B$ can be added. The assumption under which $B$ is derived is the sum of assumptions under which formulas $A_{1}, \ldots, A_{n}$ appeared in the proof.

$\mathrm{RC} \quad$ If $\vdash_{\mathrm{CLuN}}\left(\left(C_{1} \wedge \sim C_{1}\right) \vee \cdots \vee\left(C_{m} \wedge \sim C_{m}\right)\right) \vee\left(\left(A_{1} \wedge \cdots \wedge A_{n}\right) \rightarrow B\right)$, and $A_{1}, \ldots, A_{n}$ appears in the proof, then $B$ can be added provided that no formula of the form $C_{i} \wedge \sim C_{i}$ for all $1 \leqslant i \leqslant n$ occurs as the disjunct of a minimally inconsistent disjunction in that proof. The appropriate assumption is the sum of assumptions under which formulas $A_{1}, \ldots$, $A_{n}$ appeared in the proof plus the set $\left\{C_{1}, \ldots, C_{m}\right\}$. 
RD It is obligatory that if $A$ has been inferred under an assumption of consistent behavior of a set containing a formula $B$, and the formula $B \wedge \sim B$ became a disjunct of a minimally inconsistent disjunction, then $A$ has to be deleted from the proof.

Using the above meta-rules one can formulate the notion of a proof of a given formula on the basis of a set of premises $X$. But since RD can cause the deletion of a step in a proof, the notion of final derivability has to be introduced. We say that $A$ is finally derived on the basis of premises the set $X$ iff there is a proof of $A$ on the basis of that set, in which $A$ appears under some assumption $\Delta$, and even if $A$ were deleted in some extension of the proof, it could be further extended in such a way that $A$ is derived under $\Delta$ once more.

For our purposes the notion of a model is needed. By CLuN-model we mean any valuation $v$ classical with respect to positive connectives, while in the case of negation for any $A$ the following is fulfilled: $v(A)=1$ or $v(\sim A)=1$. In the standard way we semantically define the consequence relation for $\mathbf{C L u N}$.

A CLuN-model $v$ is the ACLuN1 model of a given set $X$ iff it satisfies $X$ and the only formulas of the form $B \wedge \sim B$ valid under $v$ are disjuncts of minimally inconsistent semantical consequences of the set $X$.

Since for the logic CLuN the completeness result is valid, the word 'semantical' can be dropped in the last paragraph.

A CLuN-model $v$ is the ACLuN2 model of a given set $X$ iff it satisfies $X$ and it is a minimally inconsistent CLuN-model of $X$, i.e., there is no CLuN-model of $X$, which would have validated fewer inconsistences.

\section{Jaśkowski's Logic $\mathrm{D}_{2}$}

Jaśkowski's logic $\mathbf{D}_{\mathbf{2}}$ is a propositional logic defined with the help of the modal logic S5.

A formula $A$ is a theorem of the system $\mathbf{D}_{\mathbf{2}}$ iff it is built in the standard way with the help of ' $\leftrightarrow$ ', ' $\rightarrow$ ', ' $\wedge$ ', ' $\vee$ ' and ' $\sim$ ', and the formula $\diamond A^{\prime}$, which arises by the substitution of $C \wedge \diamond D, \diamond C \rightarrow D,(\diamond C \rightarrow D) \wedge \diamond(\diamond D \rightarrow C)$ respectively for subformulas $C \wedge D, C \rightarrow D$ and $C \leftrightarrow D$ in $\diamond A$, is the theorem of $\mathbf{S 5} .^{1}$

The notion of the theorem of $\mathbf{D}_{\mathbf{2}}$ can be expressed more accurately. Let us use the following definition of Jaśkowski's transformation:

\footnotetext{
${ }^{1}$ The Appendix contains some basic notions from the modal logic pp. 22-27.
} 
DeFinition 1. By a Jaśkowski's transformation we mean the function $(\cdot)^{\mathrm{d}}$ : For $\longrightarrow$ For $_{\mathrm{m}}$ from the set of all propositional formulas into the set of all modal propositional formulas, defined by induction for any $A \in$ For:

1. If $A$ is a propositional variable, then $A^{\mathrm{d}}=A$

2. (a) $A$ is of the form $B \vee C$, we put $A^{\mathrm{d}}=B^{\mathrm{d}} \vee C^{\mathrm{d}}$

(b) $A$ is of the form $B \wedge C$, we put $A^{\mathrm{d}}=B^{\mathrm{d}} \wedge \diamond C^{\mathrm{d}}$

(c) $A$ is of the form $B \rightarrow C$, we put $A^{\mathrm{d}}=\diamond B^{\mathrm{d}} \rightarrow C^{\mathrm{d}}$

(d) $A$ is of the form $B \leftrightarrow C$, we put $A^{\mathrm{d}}=\left(\diamond B^{\mathrm{d}} \rightarrow C^{\mathrm{d}}\right) \wedge \diamond\left(\diamond C^{\mathrm{d}} \rightarrow B^{\mathrm{d}}\right)$

(e) $A$ is of the form $\sim B$, we put $A^{\mathrm{d}}=\sim\left(B^{\mathrm{d}}\right)$.

Jaśkowski's logic defined with the help of M-fragment of the modal logic $\boldsymbol{P}$ we call set:

$$
\boldsymbol{P}^{\mathbf{J}}=\left\{A \in \text { For }: \diamond A^{\mathrm{d}} \in \boldsymbol{P}\right\}
$$

i.e., for $A \in$ For: $A \in \boldsymbol{P}^{\mathbf{J}}$ iff $\diamond A^{\mathrm{d}} \in \boldsymbol{P}$.

By the above definition we have:

$$
\mathrm{D}_{2}=\mathrm{S}^{\mathrm{J}}
$$

Connectives of conjunction, implication and equivalence can be treated as abbreviations of some modal formulas. In this sense we can call them "discussive" and denote them with the additional symbol 'd':

$$
\begin{gathered}
A \wedge_{\mathrm{d}} B:=(A \wedge B)^{\mathrm{d}} \\
A \rightarrow_{\mathrm{d}} B:=(A \rightarrow B)^{\mathrm{d}} \\
A \leftrightarrow_{\mathrm{d}} B:=(A \leftrightarrow B)^{\mathrm{d}}
\end{gathered}
$$

Now we can define the consequence relation by saying that from $A_{1}, \ldots$, $A_{n}$ a formula $A$ is derivable in the sense of $\mathbf{D}_{2}$ iff on the basis of $\diamond A_{1}^{\mathrm{d}}, \ldots$, $\diamond A_{n}^{\mathrm{d}}$ a formula $\diamond A^{\mathrm{d}}$ is $\mathbf{S 5}$-provable. ${ }^{2}$

Proposition 2. The Logic $\mathbf{D}_{\mathbf{2}}$ is closed under the Modus Ponens rule:

$$
\begin{array}{cc}
A \rightarrow B \quad A \\
B
\end{array}
$$

\footnotetext{
${ }^{2}$ It is obvious that in the case of the presented definition the addition of Gödel's rule makes no difference. See the definition 18 on page 23 .
} 
Proof. It is enough to see that the following rule is provable in $\mathbf{S 5}$ :

$$
\frac{\diamond(\diamond A \rightarrow B) \quad \diamond A}{\diamond B}
$$

Let us assume that in a given proof $\diamond(\diamond A \rightarrow B)$ and $\diamond A$ appears. By Lemma 23 from p. 24 one can derive $\diamond A \rightarrow \diamond B$, therefore by the rule of Modus Ponens (being used in S5) we get $\diamond B$.

\section{Jaśkowski's logic and inconsistency adaptive logics}

In the present section we will compare Jaśkowski' logic and inconsistency adaptive logics ACLuN1 and ACLuN2. To achieve this aim connectives of implication, conjunction and equivalence will be treated as discussive ones. Firstly, let us notice that each axiom of the logic CLuN is a theorem of the $\operatorname{logic} \mathbf{D}_{\mathbf{2}}$ : a fortiori the full positive logic is contained in $\mathbf{D}_{\mathbf{2}}$. Corollary 4 was implicite expressed already by Jaśkowski (see [11] and [12]). We present its full proof here.

TheOREm 3. Each of the following schema represents a theorem of $\mathbf{D}_{\mathbf{2}}$.

Ax1. $\quad A \rightarrow(B \rightarrow A)$

Ax2. $\quad((A \rightarrow B) \rightarrow A) \rightarrow A$

Ax3. $(A \rightarrow(B \rightarrow C)) \rightarrow((A \rightarrow B) \rightarrow(A \rightarrow C))$

Ax4. $\quad A \wedge B \rightarrow A$

Ax5. $\quad A \wedge B \rightarrow B$

Ax6. $\quad A \rightarrow(B \rightarrow A \wedge B)$

Ax7. $\quad A \rightarrow A \vee B$

Ax8. $B \rightarrow A \vee B$

Ax9. $\quad(A \rightarrow B) \rightarrow((C \rightarrow B) \rightarrow(A \vee C \rightarrow B))$

Ax10. $\quad(A \leftrightarrow B) \rightarrow(A \rightarrow B)$

Ax11. $\quad(A \leftrightarrow B) \rightarrow(B \rightarrow A)$

Ax12. $\quad(A \rightarrow B) \rightarrow((B \rightarrow A) \rightarrow(A \leftrightarrow B))$

Ax13. $\quad(A \rightarrow \sim A) \rightarrow \sim A$ 
PROOF. Using the introduced definitions of discussive connectives and the definition of Jaśkowski's logic it is enough to show that modal versions (denoted by ' $A \mathrm{x}_{\mathrm{d}}$ ') of considered axioms are theorems of $\mathbf{S 5}$.

$A d \mathrm{Ax}_{\mathrm{d}} 1$. On the basis of $\mathbf{S 5}$ a formula $\diamond(\diamond A \rightarrow(\diamond B \rightarrow A))$ is by Lemma 23 and extensionality equivalent to $\diamond A \rightarrow(\diamond B \rightarrow \diamond A)$. The latter is a substitution of $A \rightarrow(B \rightarrow A)$, so belongs to $\mathbf{S 5}$.

$A d \mathrm{Ax}_{\mathrm{d}} 2$. By Lemma 23 the following equivalences hold in $\mathbf{S 5}$ : $\diamond[\diamond(\diamond(\diamond A \rightarrow B) \rightarrow A) \rightarrow A], \diamond(\diamond(\diamond A \rightarrow B) \rightarrow A) \rightarrow \diamond A,(\diamond(\diamond A \rightarrow$ $B) \rightarrow \diamond A) \rightarrow \diamond A,((\diamond A \rightarrow \diamond B) \rightarrow \diamond A) \rightarrow \diamond A$. The latter formula is a substitution of the law of Peirce. Therefore Ax2 $\in \mathbf{D}_{\mathbf{2}}$.

Ad $\mathrm{Ax}_{\mathrm{d}} 3$. The axiom $\diamond[\diamond(\diamond A \rightarrow(\diamond B \rightarrow C)) \rightarrow(\diamond(\diamond A \rightarrow B) \rightarrow$ $(\diamond A \rightarrow C))$ ] belongs to $\mathbf{S 5}$, since by Lemma 23 it is equivalent to the formula $(\diamond A \rightarrow(\diamond B \rightarrow \diamond C)) \rightarrow((\diamond A \rightarrow \diamond B) \rightarrow(\diamond A \rightarrow \diamond C))$. The latter is a substitution of Frege's law.

$A d \mathrm{Ax}_{\mathrm{d}} 4 . \vdash_{\mathrm{S} 5} \diamond(\diamond(A \wedge \diamond B) \rightarrow A)$ by lemmas 23 and 24 , this formula is equivalent to the law of absorption for conjunction.

$A d \mathrm{Ax}_{\mathrm{d}} 5 . \vdash_{\mathrm{S} 5} \diamond(\diamond(A \wedge \diamond B) \rightarrow B)$. The proof proceeds in an analogue way.

$A d \mathrm{Ax}_{\mathrm{d}} 6 . \vdash_{\mathrm{S} 5} \diamond(\diamond A \rightarrow(\diamond B \rightarrow A \wedge \diamond B))$. The proof goes similarly since the formula under consideration is equivalent to the law of adjunction.

Ad $\mathrm{Ax}_{\mathrm{d}} 7 . \vdash_{\mathrm{S} 5} \diamond(\diamond A \rightarrow A \vee B)$ This follows directly from the law of absorption for disjunction, Lemma 23 and the monotonicity rule.

$A d \mathrm{Ax}_{\mathrm{d}} 8 . \vdash_{\mathrm{S} 5} \diamond(\diamond B \rightarrow A \vee B)$ as in the previous case.

$A d \mathrm{Ax}_{\mathrm{d}} 9 . \vdash_{\mathrm{S} 5} \diamond(\diamond(\diamond A \rightarrow B) \rightarrow(\diamond(\diamond C \rightarrow B) \rightarrow(\diamond(A \vee C) \rightarrow B)))$. By Lemma 23 this formula is equivalent to $(\diamond A \rightarrow \diamond B) \rightarrow((\diamond C \rightarrow \diamond B) \rightarrow$ $(\diamond(A \vee C) \rightarrow \diamond B)$ ), while by regularity (t3 on p. 23$)$ it is equivalent to the substitution of: $(A \rightarrow B) \rightarrow((C \rightarrow B) \rightarrow(A \vee C \rightarrow B))$.

$A d \mathrm{Ax}_{\mathrm{d}} 10 . \quad \vdash_{\mathrm{S} 5} \diamond[\diamond((\diamond A \rightarrow B) \wedge \diamond(\diamond B \rightarrow A)) \rightarrow(\diamond A \rightarrow B)]$. By lemmas 23 and 24 this formula is equivalent to: $((\diamond A \rightarrow \diamond B) \wedge(\diamond B \rightarrow$ $\diamond A)) \rightarrow(\diamond A \rightarrow \diamond B)$, which is a substitution of the law of the absorption for conjunction.

$A d \mathrm{Ax}_{\mathrm{d}} 11 . \vdash_{\mathrm{S} \mathbf{5}} \diamond[\diamond((\diamond A \rightarrow B) \wedge \diamond(\diamond B \rightarrow A)) \rightarrow(\diamond B \rightarrow A)]$. The reason is the same as above.

$A d \mathrm{Ax}_{\mathrm{d}} 12 . \quad \vdash_{\mathrm{S} \mathbf{5}} \diamond[\diamond(\diamond A \rightarrow B) \rightarrow(\diamond(\diamond B \rightarrow A) \rightarrow((\diamond A \rightarrow B) \wedge$ $\diamond(\diamond A \rightarrow B)))]$. This formula is equivalent to the substitution of the law of adjunction: $(\diamond A \rightarrow \diamond B) \rightarrow[(\diamond B \rightarrow \diamond A) \rightarrow((\diamond A \rightarrow \diamond B) \wedge(\diamond A \rightarrow \diamond B))]$.

$A d \mathrm{Ax}_{\mathrm{d}} 13 . \vdash_{\mathrm{S} \mathbf{5}} \diamond(\diamond(\diamond A \rightarrow \sim A) \rightarrow \sim A)$. By Lemma 23 and condition (1) from page 22 of inter-definability of ' $\square$ ' and ' $\diamond$ ' it is equivalent to $(\diamond A \rightarrow$ 
$\sim \square A) \rightarrow \sim \square A$, and by the disjunctive syllogism it is equivalent to the schema $(\sim \diamond A \vee \sim \square A) \rightarrow \sim \square A$. The last one is a theorem of the logic S5 by the contraposition of the axiom $D: \sim \diamond A \rightarrow \sim \square A$. (MP) and the substitution of $(A \rightarrow B) \rightarrow(A \vee B \rightarrow B)$.

As a consequence of the last theorem and Proposition 2 we obtain:

Corollary 4. The logic CLuN is contained in $\mathbf{D}_{\mathbf{2}}$.

However, the analogous observation does not hold for adaptive logics built on CLuN as the lower limit logic:

Proposition 5. The adaptive logics ACLuN1 and ACLuN2 are crossing with $\mathbf{D}_{\mathbf{2}}$.

Proof. Since for any set of formulas $X$ and a formula $A$ holds $X \vdash_{C L u N}$ $A \Rightarrow X \vdash_{\text {ACLuN1 }} A \Rightarrow X \vdash_{\text {CL }} A, X \vdash_{\text {CLuN }} A \Rightarrow X \vdash_{\text {ACLuN2 }} A \Rightarrow X \vdash_{\text {CL }} A$ (see $[2,3]$ ) and simultaneously, if $X$ is a consistent set, then $X \vdash_{\text {ACLuN1 }}$ $A \Leftrightarrow X \vdash_{\text {ACLuN2 }} A \Leftrightarrow X \vdash_{\text {CL }} A$, therefore $\{p, \sim p \vee q\} \vdash_{\text {ACLuN1 }} q$ and $\{p, \sim p \vee q\} \vdash_{\text {ACLuN2 }} q$. It is easy to see, that $\{p, \sim p \vee q\} \nvdash_{\mathbf{D}_{\mathbf{2}}} q$. Indeed $\{\diamond p, \diamond \sim p \vee \diamond q\} \nvdash_{\mathbf{S} 5} \diamond q$ since $\{\diamond p, \diamond \sim p \vee \diamond q\} \forall_{\mathbf{S} 5} \diamond q$ :

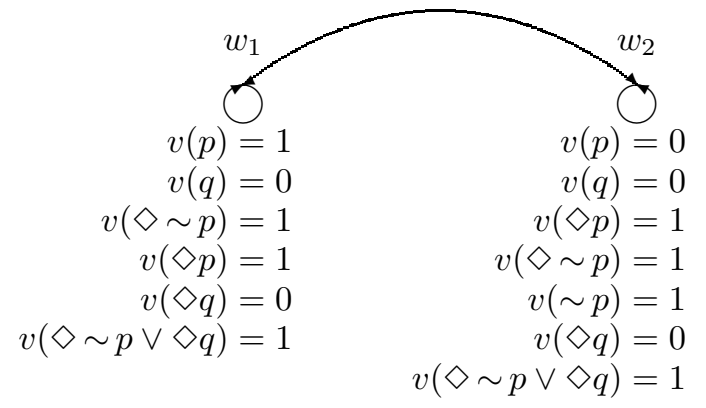

On the other hand, one can observe that $\{p, \sim p\} \nvdash_{\text {ACluN2 }} \sim(p \wedge \sim p)$. Indeed, minimally inconsistent $\mathbf{C L u N}$-models of our premises do not validate the formula $\sim(p \wedge \sim p)$ (the formula $\{p \wedge \sim p\}$ is a CLuN consequence of the set of our premises and there is no $\mathbf{C L u N}$-model of our premises which would validate fewer inconsistencies). Since in general $X \vdash_{A C L u N 1} A \Rightarrow X \vdash_{\text {ACLuN2 }}$, the same observation holds for the ACLuN1 consequence: $\{p, \sim p\} \nvdash_{\text {ACLuN1 }}$ $\sim(p \wedge \sim p)$. Of course $\{p, \sim p\} \vdash_{\mathbf{D}_{\mathbf{2}}} \sim(p \wedge \sim p)$. In fact, $\diamond \sim(p \wedge \diamond \sim p)$ is equivalent to formula $\diamond(p \rightarrow \square p)$, which belongs to $\mathbf{S 5}$.

Let us notice that $\{p \wedge \sim p, p \vee \sim(p \wedge \sim p)\} \nvdash_{\text {ACLuN1 }} \sim(p \wedge \sim p)$. Since on the basis of the premises one can unconditionally derive $p \wedge \sim p$, while only assuming consistent behavior of $p$ one can derive $\sim(p \wedge \sim p)$. 
Lemma 6. Let $\mathbf{K D}^{*} \mathbf{T}^{*}$ is the normal modal logic arising by extension of the logic $\mathbf{K}$ with the axioms:

$\left(\mathrm{T}^{*}\right) \quad \square \diamond \diamond A \rightarrow \diamond A$, equivalently $\diamond(\diamond \diamond A \rightarrow A)$

$\left(\mathrm{D}^{*}\right) \quad \square \diamond A \rightarrow \diamond A$, equivalently $\diamond(\diamond A \rightarrow A)$

In $\mathbf{K D}^{*} \mathbf{T}^{*}$ the following formulas are provable:

$$
\begin{gathered}
\square A \rightarrow \diamond A \\
\diamond(\square \diamond(A \vee B) \rightarrow(\square \diamond A \vee \square \diamond B)) \\
\diamond[\diamond(\diamond A \rightarrow B) \rightarrow(\diamond(\diamond C \rightarrow B) \rightarrow(\diamond(A \vee C) \rightarrow B))] \\
\square A \rightarrow \diamond \square(\square A \wedge A) \\
\square \diamond(\square A \vee A) \rightarrow \diamond A
\end{gathered}
$$

Proof. Ad (D)

1. $(\diamond A \rightarrow A) \rightarrow(A \rightarrow A)$

2. $\diamond(\diamond A \rightarrow A) \rightarrow \diamond(A \rightarrow A)$

3. $\diamond(A \rightarrow A)$ $A d(\mathrm{DN} 1)$

1. $\square \diamond(A \vee B) \rightarrow \square \diamond A \vee \diamond \diamond B$

2. $\square \diamond A \vee \diamond \diamond B \rightarrow \diamond \diamond B \vee \square \diamond A$

3. $\square \diamond(A \vee B) \rightarrow \diamond \diamond B \vee \square \diamond A$

4. $\square \square \diamond(A \vee B) \rightarrow \square \diamond \diamond B \vee \diamond \square \diamond A$

5. $\square \diamond \diamond B \vee \diamond \square \diamond A \rightarrow \diamond \square \diamond A \vee \square \diamond \diamond B$

6. $\square \square \diamond(A \vee B) \rightarrow \diamond \square \diamond A \vee \square \diamond \diamond B$

7. $\square \diamond \diamond B \rightarrow \diamond \square \square \diamond \diamond B$ the substitution to th

8. $\diamond \square \square \diamond \diamond B \rightarrow \diamond \square \diamond B \quad$ the axiom $\left(\mathrm{T}^{*}\right)$ and monotonicity rules

9. $\square \diamond \diamond B \rightarrow \diamond \square \diamond B$

10. $\diamond \square \diamond A \vee \square \diamond \diamond B \rightarrow \diamond \square \diamond A \vee \diamond \square \diamond B$ the substitution of $(A \rightarrow B) \rightarrow((C \vee A) \rightarrow(C \vee B)),(\mathrm{MP})$ and 9

11. $\square \square \diamond(A \vee B) \rightarrow \diamond \square \diamond A \vee \diamond \square \diamond B$

12. $\diamond \square \diamond A \vee \diamond \square \diamond B \rightarrow \diamond(\square \diamond A \vee \square \diamond B)$

13. $\square \square \diamond(A \vee B) \rightarrow \diamond(\square \diamond A \vee \square \diamond B)$

14. $\diamond(\square \diamond(A \vee B) \rightarrow(\square \diamond A \vee \square \diamond B))$
(MP), the law of identity, and $(A \rightarrow A) \rightarrow((\diamond A \rightarrow A) \rightarrow(A \rightarrow A))$ 1 and the monotonicity rule (MP), 2 and ( $\left.\mathrm{D}^{*}\right)$

t3, monotonicity, and t 4 commutativity of ' $\mathrm{V}$ ' the law of syllogism, 1 and 2 3 , monotonicity rule and $\mathbf{t} 4$ commutativity of ' $\mathrm{V}$ ' the law of syllogism, 4 and 5 the law of syllogism, 7 and 8 the law of syllogism, 6 and 10 t3 the law of syllogism, 11 and 12 13 and $\mathbf{t} 5$ 
$A d(\mathrm{DN} 2)$

1. $(\square \diamond A \rightarrow \diamond B) \rightarrow\{(\square \diamond C \rightarrow \diamond B) \rightarrow[(\square \diamond(A \vee C) \rightarrow(\square \diamond A \vee \square \diamond C)) \rightarrow$ $\rightarrow(\square \diamond(A \vee C) \rightarrow \diamond B)]\} \quad$ the substitution of $(A \rightarrow B) \rightarrow\{[(C \rightarrow B) \rightarrow((D \rightarrow A \vee C) \rightarrow(D \rightarrow B))\}$

2. $\square \diamond(\diamond A \rightarrow B) \rightarrow \diamond(\diamond A \rightarrow B) \quad$ the axiom $\left(\mathrm{D}^{*}\right): A /(\diamond A \rightarrow B)$

3. $\square(\square \diamond A \rightarrow \diamond B) \rightarrow(\square \diamond A \rightarrow \diamond B) \quad 2$ and $\mathbf{t} 5$

4. $\square \diamond(\diamond C \rightarrow B) \rightarrow \diamond(\diamond C \rightarrow B) \quad$ the axiom $\left(\mathrm{D}^{*}\right): A /(\diamond C \rightarrow B)$

5. $\square(\square \diamond C \rightarrow \diamond B) \rightarrow(\square \diamond C \rightarrow \diamond B) \quad 4$ and t 5

6. $\square(\square \diamond A \rightarrow \diamond B) \rightarrow\{\square(\square \diamond C \rightarrow \diamond B) \rightarrow$

$\rightarrow[(\square \diamond(A \vee C) \rightarrow(\square \diamond A \vee \square \diamond C)) \rightarrow(\square \diamond(A \vee C) \rightarrow \diamond B)]\}$

the commutativity rule, the law of syllogism, 5,3 and 1

7. $(\square \diamond(A \vee C) \rightarrow(\square \diamond A \vee \square \diamond C)) \rightarrow$

$\rightarrow\{\square(\square \diamond A \rightarrow \diamond B) \rightarrow[\square(\square \diamond C \rightarrow \diamond B) \rightarrow(\square \diamond(A \vee C) \rightarrow \diamond B)]\}$

8. $\diamond(\square \diamond(A \vee C) \rightarrow(\square \diamond A \vee \square \diamond C)) \rightarrow$

the commutativity rule and 6

$\rightarrow \diamond\{\square(\square \diamond A \rightarrow \diamond B) \rightarrow[\square(\square \diamond C \rightarrow \diamond B) \rightarrow(\square \diamond(A \vee C) \rightarrow \diamond B)]\}$ monotonicity rule and 7

9. $\diamond\{\square(\square \diamond A \rightarrow \diamond B) \rightarrow[\square(\square \diamond C \rightarrow \diamond B) \rightarrow(\square \diamond(A \vee C) \rightarrow \diamond B)]\}$

$(\mathrm{MP}), 8$ and (DN1)

10. $\diamond[\square \diamond(\diamond A \rightarrow B) \rightarrow[\square \diamond(\diamond C \rightarrow B) \rightarrow \diamond(\diamond(A \vee C) \rightarrow B)]]$

9 , laws of extensionality and $\mathbf{t} 5$

11. $\diamond \diamond[\diamond(\diamond A \rightarrow B) \rightarrow[\diamond(\diamond C \rightarrow B) \rightarrow(\diamond(A \vee C) \rightarrow B)]]$

10 , extensionality and $\mathbf{t} 5$

12. $\square \diamond \diamond[\diamond(\diamond A \rightarrow B) \rightarrow[\diamond(\diamond C \rightarrow B) \rightarrow(\diamond(A \vee C) \rightarrow B)]]$

13. $\diamond[\diamond(\diamond A \rightarrow B) \rightarrow[\diamond(\diamond C \rightarrow B) \rightarrow(\diamond(A \vee C) \rightarrow B)]]$

11 and $(\mathrm{RG})$

the axiom $\left(\mathrm{T}^{*}\right), 12$ and $(\mathrm{MP})$

Ad (DN3) We will prove an auxiliary theorem:

$\left(\mathrm{DN} 3^{\prime}\right)$

$\square \diamond(\diamond A \vee A) \rightarrow \diamond A$

1. $\diamond[\diamond(\diamond \diamond A \rightarrow A) \rightarrow(\diamond(\diamond A \rightarrow A) \rightarrow(\diamond(\diamond A \vee A) \rightarrow A))]$

the substitution of (DN2): $A / \diamond A, B / A$ and $C / A$

2. $\square(\square \diamond \diamond A \rightarrow \diamond A) \rightarrow[\square(\square \diamond A \rightarrow \diamond A) \rightarrow(\square \diamond(\diamond A \vee A) \rightarrow \diamond A)]$

3. $\square(\square \diamond \diamond A \rightarrow \diamond A)$

1 , extensionality and $\mathbf{t} 5$

4. $\square(\square \diamond A \rightarrow \diamond A)$

$\left(\mathrm{T}^{*}\right)$ and $(\mathrm{RG})$

5. $\square \diamond(\diamond A \vee A) \rightarrow \diamond A$

$\left(\mathrm{D}^{*}\right)$ and $(\mathrm{RG})$

(MP), 2, 3 and 4 
Using the rule of contraposition to $\left(\mathrm{DN} 3^{\prime}\right)$, where $\sim A$ is substituted for $A$ we get: $\sim \diamond \sim A \rightarrow \sim \square \diamond(\diamond \sim A \vee \sim A)$. (DN3) is arising by the condition (1), via de Morgan's law:

6. $\square \diamond(\diamond \neg A \vee \neg A) \rightarrow \diamond \neg A \quad$ contraposition of the substitution of (DN3')

7. $\square A \rightarrow \diamond \square(\square A \wedge A) \quad 6$, contraposition, de Morgan's law and (1) Ad (DN4)

1. $(\square A \vee A) \rightarrow(\diamond A \vee A)$

2. $\square \diamond(\square A \vee A) \rightarrow \square \diamond(\diamond A \vee A)$

the axiom $\mathrm{D}$ and addition of a new disjunct to arguments of an implication 1 and the monotonicity rules

3. $\square \diamond(\diamond A \vee A) \rightarrow \diamond A$

$\left(\mathrm{DN} 3^{\prime}\right)$

4. $\square \diamond(\square A \vee A) \rightarrow \diamond A$

the law of syllogism, 2 and 3

Theorem 7. The minimal normal logic, which contains axioms $\diamond(\mathrm{Ax} 1)^{\mathrm{d}_{-}}$ $\diamond(\operatorname{Ax} 13)^{\mathrm{d}}$, closed with respect to the rule:

$$
\frac{\diamond A \quad \diamond(\diamond A \rightarrow B)}{\diamond B}
$$

is the logic $\mathbf{K} \mathbf{D}^{*} \mathbf{T}^{*}$, i.e., $\mathbf{K D}^{*} \mathbf{T}^{*}=\mathbf{J}(\mathbf{C L u N})$

Proof. Firstly, we will show that each of axioms $\mathrm{Ax}_{\mathrm{d}} 1-\mathrm{Ax}_{\mathrm{d}} 13$ is provable in $\mathbf{K D}^{*} \mathbf{T}^{*}$.

Ad $\mathrm{Ax}_{\mathrm{d}} 1$.

1. $\square \diamond A \rightarrow \diamond A$

2. $\square \diamond B \rightarrow(\square \diamond A \rightarrow \diamond A)$

3. $\square \diamond A \rightarrow(\square \diamond B \rightarrow \diamond A)$

1 and the classical logic

4. $\diamond(\diamond A \rightarrow(\diamond B \rightarrow A))$ 2 and the commutativity law

$3, \mathbf{t} 5$ and extensionality rule

$A d \mathrm{Ax}_{\mathrm{d}} 2$. Let us start with the observation, that in the considered logic the following theorem is provable:

$\left(\mathrm{DN} 4^{\prime}\right)$

$\square \diamond(\square \diamond A \vee A) \rightarrow \diamond A$

Indeed:

1. $\square \diamond A \rightarrow \diamond A$

2. $\square \diamond A \vee A \rightarrow \diamond A \vee A$

1 and the addition of a new

3. $\square \diamond(\square \diamond A \vee A) \rightarrow \square \diamond(\diamond A \vee A)$ disjunct to arguments of an implication

4. $\square \diamond(\diamond A \vee A) \rightarrow \diamond A$

5. $\square \diamond(\square \diamond A \vee A) \rightarrow \diamond A$

2, monotonicity rules

$\left(\mathrm{DN}^{\prime}\right)$

3,4 and the law of syllogism 
Using (DN4 $4^{\prime}$ ) we prove $\mathrm{Ax}_{\mathrm{d}} 2$.

1. $((\square \diamond A \wedge \sim \diamond B) \vee A) \rightarrow(\square \diamond A \vee A) \quad$ the law of absorption and the addition of a new disjunct to arguments of an implication

2. $\square \diamond((\square \diamond A \wedge \sim \diamond B) \vee A) \rightarrow \square \diamond(\square \diamond A \vee A) \quad 1$ and monotonicity rules

3. $\square \diamond((\square \diamond A \wedge \sim \diamond B) \vee A) \rightarrow \diamond A$

$2,\left(\mathrm{DN} 4^{\prime}\right)$

4. $\diamond[\diamond((\square \diamond A \wedge \sim \diamond B) \vee A) \rightarrow A]$

5. $\diamond[\diamond(\sim(\square \diamond A \wedge \sim \diamond B) \rightarrow A) \rightarrow A]$

6. $\diamond[\diamond((\square \diamond A \rightarrow \diamond B) \rightarrow A) \rightarrow A]$

7. $\diamond[\diamond(\diamond(\diamond A \rightarrow B) \rightarrow A) \rightarrow A]$ and the law of the syllogism 3 and $\mathbf{t} 5$ 4, extensionality and the disjunctive syllogism 5 , extensionality and the law of negation of ' $\rightarrow$ '

Ad $\mathrm{Ax}_{\mathrm{d}} 3$.

1. $(\square \diamond A \wedge \square \sim C) \rightarrow \square(\diamond A \wedge \sim C)$ the regularity condition $\mathbf{t} 2$

2. $(\square(\square \diamond A \rightarrow \diamond B) \wedge(\square \diamond A \wedge \square \sim C)) \rightarrow(\square(\square \diamond A \rightarrow \diamond B) \wedge \square(\diamond A \wedge \sim C))$ 1 and the the addition of a new conjunct to arguments of an implication

3. $(\square(\square \diamond A \rightarrow \diamond B) \wedge \square(\diamond A \wedge \sim C)) \rightarrow \square((\square \diamond A \rightarrow \diamond B) \wedge(\diamond A \wedge \sim C))$

4. $(\square(\square \diamond A \rightarrow \diamond B) \wedge(\square \diamond A \wedge \square \sim C)) \rightarrow$ $\rightarrow \square((\square \diamond A \rightarrow \diamond B) \wedge(\diamond A \wedge \sim C)) \quad$ the law of syllogism, 2. and 3.

5. $\square((\square \diamond A \rightarrow \diamond B) \wedge(\diamond A \wedge \sim C)) \rightarrow \diamond \square[\square((\square \diamond A \rightarrow \diamond B) \wedge(\diamond A \wedge \sim C))$ $\wedge((\square \diamond A \rightarrow \diamond B) \wedge(\diamond A \wedge \sim C))]$

$(\mathrm{DN} 3): A /[(\square \diamond A \rightarrow \diamond B) \wedge(\diamond A \wedge \sim C)]$

6. $\square((\square \diamond A \rightarrow \diamond B) \wedge(\diamond A \wedge \sim C)) \rightarrow \square \diamond A \quad$ the absorption law and the monotonicity rule

7. $[\square((\square \diamond A \rightarrow \diamond B) \wedge(\diamond A \wedge \sim C)) \wedge((\square \diamond A \rightarrow \diamond B) \wedge(\diamond A \wedge \sim C))] \rightarrow$ $\rightarrow[\square \diamond A \wedge((\square \diamond A \rightarrow \diamond B) \wedge(\diamond A \wedge \sim C))] \quad 6$ and the the addition of a new conjunct to arguments of an implication

8. $\diamond \square[\square((\square \diamond A \rightarrow \diamond B) \wedge(\diamond A \wedge \sim C)) \wedge((\square \diamond A \rightarrow \diamond B) \wedge(\diamond A \wedge \sim C))] \rightarrow$ $\rightarrow \diamond \square[\square \diamond A \wedge((\square \diamond A \rightarrow \diamond B) \wedge(\diamond A \wedge \sim C))]$

9. $\square \diamond A \wedge(\square \diamond A \rightarrow \diamond B) \rightarrow \diamond B$

7 and the monotonicity rules the substitution of the principle of detachment

10. $[\square \diamond A \wedge((\square \diamond A \rightarrow \diamond B) \wedge(\diamond A \wedge \sim C))] \rightarrow(\diamond A \wedge(\diamond B \wedge \sim C))$

9 and the the addition of a new conjunct to arguments of an implication, and laws of associativity and commutativity of conjunction

11. $\diamond \square[\square \diamond A \wedge((\square \diamond A \rightarrow \diamond B) \wedge(\diamond A \wedge \sim C))] \rightarrow \diamond \square[\diamond A \wedge(\diamond B \wedge \sim C)]$ 
10 and the monotonicity rules

12. $\diamond \square[\diamond A \wedge(\diamond B \wedge \sim C)] \rightarrow \diamond[\square \diamond A \wedge(\square \diamond B \wedge \square \sim C)]$

extensionality and regularity

13. $(\square(\square \diamond A \rightarrow \diamond B) \wedge(\square \diamond A \wedge \square \sim C)) \rightarrow \diamond(\square \diamond A \wedge(\square \diamond B \wedge \square \sim C))$ the law of syllogism $4,5,8,11$ and 12

14. $\sim \diamond(\square \diamond A \wedge(\square \diamond B \wedge \square \sim C)) \rightarrow \sim[\square(\square \diamond A \rightarrow \diamond B) \wedge(\square \diamond A \wedge \square \sim C)]$ 13, contraposition

15. $\square[\square \diamond A \rightarrow(\square \diamond B \rightarrow \diamond C)] \rightarrow[\square(\square \diamond A \rightarrow \diamond B) \rightarrow(\square \diamond A \rightarrow \diamond C)]$

14 , the law of negation of ' $\rightarrow$ ' and condition (1)

16. $\square[\square \diamond A \rightarrow \diamond(\diamond B \rightarrow C)] \rightarrow[\square \diamond(\diamond A \rightarrow B) \rightarrow \diamond(\diamond A \rightarrow C)]$

15 and $\mathbf{t} 5$

17. $\diamond[\diamond(\diamond A \rightarrow(\diamond B \rightarrow C)) \rightarrow(\diamond(\diamond A \rightarrow B) \rightarrow(\diamond A \rightarrow C))]$

16 and $\mathbf{t} 5$

Ad $\mathrm{Ax}_{\mathrm{d}} 4$.

1. $A \wedge \diamond B \rightarrow A$

2. $\square \diamond(A \wedge \diamond B) \rightarrow \square \diamond A$

3. $\square \diamond A \rightarrow \diamond A$

the absorption law

4. $\square \diamond(A \wedge \diamond B) \rightarrow \diamond A$

5. $\diamond(\diamond(A \wedge \diamond B) \rightarrow A)$

the law of syllogism, 2 and 3

4 and $\mathbf{t} 5$

Ad $\mathrm{Ax}_{\mathrm{d}} 5$.

1. $A \wedge \diamond B \rightarrow \diamond B$

2. $\square \diamond(A \wedge \diamond B) \rightarrow \square \diamond \diamond B$

the absorption law

3. $\square \diamond \diamond B \rightarrow \diamond B$

1 and monotonicity rules

4. $\square \diamond(A \wedge \diamond B) \rightarrow \diamond B$

$\left(\mathrm{T}^{*}\right)$

5. $\diamond(\diamond(A \wedge \diamond B) \rightarrow B)$

the law of syllogism, 2 and 3

Ad $\mathrm{Ax}_{\mathrm{d}} 6$.

1. $A \rightarrow(\diamond B \rightarrow A \wedge \diamond B)$

4 and $\mathbf{t} 5$

2. $\diamond A \rightarrow \diamond(\diamond B \rightarrow A \wedge \diamond B)$

the law of adjunction

3. $\square \diamond A \rightarrow \diamond A$

4. $\square \diamond A \rightarrow \diamond(\diamond B \rightarrow A \wedge \diamond B)$

5. $\diamond(\diamond A \rightarrow(\diamond B \rightarrow A \wedge \diamond B))$

1 and the monotonicity rule

the law of syllogism, 3 and 2

Ad $\mathrm{Ax}_{\mathrm{d}} 7$.

1. $\diamond A \rightarrow \diamond(A \vee B) \quad$ the absorption law and the monotonicity rule 
2. $\square \diamond A \rightarrow \diamond A$

3. $\square \diamond A \rightarrow \diamond(A \vee B)$

the law of syllogism, 2 and 1

4. $\diamond(\diamond A \rightarrow A \vee B)$

3 and $\mathbf{t} 5$

$A d \mathrm{Ax}_{\mathrm{d}} 8$. I.e., $\diamond(\diamond B \rightarrow A \vee B)$. The proof is analogous.

$A d \mathrm{Ax}_{\mathrm{d}}$ 9. I.e., $\diamond[\diamond(\diamond A \rightarrow B) \rightarrow(\diamond(\diamond C \rightarrow B) \rightarrow(\diamond(A \vee C) \rightarrow B))]$. This is just the formula (DN2) from Lemma DN1.

Ad $\mathrm{Ax}_{\mathrm{d}} 10$.

1. $\diamond[\diamond((\diamond A \rightarrow B) \wedge \diamond(\diamond B \rightarrow A)) \rightarrow(\diamond A \rightarrow B)]$

$$
\mathrm{Ax}_{\mathrm{d}} 4, A /(\diamond A \rightarrow B), B /(\diamond B \rightarrow A)
$$

Ad $\mathrm{Ax}_{\mathrm{d}} 11$.

1. $\diamond[\diamond((\diamond A \rightarrow B) \wedge \diamond(\diamond B \rightarrow A)) \rightarrow(\diamond B \rightarrow A)]$

$$
\mathrm{Ax}_{\mathrm{d}} 5, A /(\diamond A \rightarrow B), B /(\diamond B \rightarrow A)
$$

Ad $\mathrm{Ax}_{\mathrm{d}} 12$.

1. $\diamond[\diamond(\diamond A \rightarrow B) \rightarrow[\diamond(\diamond B \rightarrow A) \rightarrow((\diamond A \rightarrow B) \wedge \diamond(\diamond B \rightarrow A))]]$

$$
\mathrm{Ax}_{\mathrm{d}} 6, A /(\diamond A \rightarrow B), B /(\diamond B \rightarrow A)
$$

Ad $\mathrm{Ax}_{\mathrm{d}} 13$.

1. $\square \diamond(\square \sim A \vee \sim A) \rightarrow \diamond \sim A$

2. $\square \diamond(\sim \square \sim A \rightarrow \sim A) \rightarrow \diamond \sim A$

(DN4): $A / \sim A$

3. $\diamond(\diamond(\diamond A \rightarrow \sim A) \rightarrow \sim A)$

the disjunctive syllogism and the extensionality t5 and the condition (1)

Let us assume that $\diamond A \in \mathbf{K D}^{*} \mathbf{T}^{*}$ and $\diamond(\diamond A \rightarrow A) \in \mathbf{K D}^{*} \mathbf{T}^{*}$. By (RG) and $\mathbf{t} 5$ we have that $\square \diamond A \in \mathbf{K D}^{*} \mathbf{T}^{*}$ and $\square \diamond A \rightarrow \diamond A \in \mathbf{K} \mathbf{D}^{*} \mathbf{T}^{*}$. So, by (MP) we have $\diamond A \in \mathbf{K D}^{*} \mathbf{T}^{*}$.

In the opposite direction, it is easy to see that axioms $\left(\mathrm{D}^{*}\right)$ and $\left(\mathrm{T}^{*}\right)$ cannot be omitted by postulated minimality - indeed these axioms are just appropriate substitutions of some the axioms $\mathrm{Ax}_{\mathrm{d}} 1-\mathrm{Ax}_{\mathrm{d}} 13$ :

Ad $\mathrm{T}^{*}$.

1. $\diamond(\diamond(\diamond A \wedge \diamond A) \rightarrow A)$

2. $\diamond(\diamond \diamond A \rightarrow A)$

3. $\square \diamond \diamond A \rightarrow \diamond A$

the substitution of $\mathrm{Ax}_{\mathrm{d}} 5: A / \diamond A$ and $B / A$

1 , idempotency and extensionality

2 and $\mathbf{t} 5$

Ad $\mathrm{D}^{*}$.

1. $\diamond(\diamond A \rightarrow(\diamond A \rightarrow A))$

the substitution of $\mathrm{Ax}_{\mathrm{d}} 1: B / A$ 
2. $\diamond(\diamond A \rightarrow A) \quad$ 1, the substitution of $(A \rightarrow(A \rightarrow B)) \leftrightarrow(A \rightarrow B)$

3. $\square \diamond A \rightarrow \diamond A$ and extensionality 2 and $\mathbf{t} 5$

Lemma 8. The axiom $\left(\mathrm{T}^{*}\right)$ is equivalent to the formula $\square A \rightarrow \diamond \square \square A$.

Proof. obvious: contraposition of the substitution of $\left(\mathrm{T}^{*}\right): A / \sim A$ and the condition (1).

Lemma 9. The axiom $\left(\mathrm{D}^{*}\right)$ is equivalent to the formula $\square A \rightarrow \diamond \square A$.

Proof. is analogous to the previous one.

Let us recall:

Theorem 10 (Furmanowski [7]). $\diamond \mathbf{S} \mathbf{4}=\diamond \mathbf{S 5}$.

The following logic is formulated by Perzanowski ([14]).

Definition 11. $\mathbf{S} \mathbf{5}_{\mathbf{M}}$ is the normal logic defined with the help of following axioms:

$$
\begin{gathered}
\square A \rightarrow \diamond A \\
\diamond \square(\diamond \square A \rightarrow \square A) \\
\diamond \square(\square A \rightarrow A)
\end{gathered}
$$

with the additional rule

$$
\frac{\diamond \diamond A}{\diamond A}
$$

Theorem 10 follows from the stronger theorem:

Theorem 12 (Perzanowski [14]). S5 $\mathbf{M}$ is the minimal normal logic for which the set of all its theorems which start with ' $\diamond$ ' is equal to $\diamond \mathbf{S 5}$.

We'll prove this theorem, simplifying Perzanowski's axioms. Let us notice that in [14] Perzanowski gave the general method of axiomatization of $M$ fragments of normal logics, not only of S5.

Firstly we show that Perzanowski's system is equivalent the system introduced in Lemma 6. The theorem by Dziobiak is presented here with the full proof:

Theorem 13 (Dziobiak [4]). S5 $\mathbf{M}=\mathbf{K D}^{*} \mathbf{T}^{*}$ 
Proof. We show that using given axiomatization one can prove specific axioms and the rule of the logic $\mathbf{S} \mathbf{5}_{\mathbf{M}}$. Let us notice that:

Ad (MT)

1. $\sim \diamond \square(\square A \rightarrow A) \rightarrow \square \diamond(\square A \wedge \sim A)$

the law of negation of ' $\rightarrow$ ' and the condition (1)

2. $\diamond(\square A \wedge \sim A) \rightarrow \diamond \square A \wedge \diamond \sim A$

t6: $A / \square A, B / \sim A$

3. $\square \diamond(\square A \wedge \sim A) \rightarrow \square(\diamond \square A \wedge \diamond \sim A)$

4. $\square(\diamond \square A \wedge \diamond \sim A) \rightarrow \sim \diamond(\diamond \square A \rightarrow \square A)$

2 and the monotonicity rule the law of negation of ' $\rightarrow$ ' and condition (1)

5. $\sim \diamond \square(\square A \rightarrow A) \rightarrow \sim \diamond(\diamond \square A \rightarrow \square A)$ the law of syllogism and 1,3 and 4

6. $\diamond(\diamond \square A \rightarrow \square A) \rightarrow \diamond \square(\square A \rightarrow A$ raposition of 5

7. $\square \diamond \square A \rightarrow \diamond \square A$ $\left(\mathrm{D}^{*}\right): A / \square A$

8. $(\square \diamond \square A \rightarrow \diamond \square A) \rightarrow \diamond(\diamond \square A \rightarrow \square A)$

9. $\diamond \square(\square A \rightarrow A)$

Ad (M5)

1. $\sim \diamond \diamond \square(\diamond \square A \rightarrow \square A) \rightarrow \square \square \diamond(\diamond \square A \wedge \sim \square A)$

the law of negation of ' $\rightarrow$ ' and condition (1)

2. $\square \square \diamond(\diamond \square A \wedge \sim \square A) \rightarrow(\square \square \diamond \diamond \square A \wedge \sim \diamond \diamond \square \square A)$ t6: $A /(\diamond \square A)$, $B /(\sim \square A)$, the monotonicity rule and condition (1)

3. $(\square \square \diamond \diamond \square A \wedge \sim \diamond \diamond \square \square A) \rightarrow \sim(\square \square \diamond \diamond \square A \rightarrow \diamond \diamond \square \square A)$

the law of negation of ' $\rightarrow$ '

4. $\sim \diamond \diamond \square(\diamond \square A \rightarrow \square A) \rightarrow \sim(\square \square \diamond \diamond \square A \rightarrow \diamond \diamond \square \square A)$ the law of syllogism, 1, 2 and 3

$5(\sim \diamond \diamond \square(\diamond \square A \rightarrow \square A) \rightarrow \sim(\square \square \diamond \diamond \square A \rightarrow \diamond \diamond \square \square A)) \rightarrow$ $\rightarrow((\square \square \diamond \diamond \square A \rightarrow \diamond \diamond \square \square A) \rightarrow \diamond \diamond \square(\diamond \square A \rightarrow \square A))$

the law of contraposition

6. $(\square \square \diamond \diamond \square A \rightarrow \diamond \diamond \square \square A) \rightarrow \diamond \diamond \square(\diamond \square A \rightarrow \square A)$

(MP), 4 and 5

7. $\square \diamond \diamond \square A \rightarrow \diamond \square A$

8. $\square \square \diamond \diamond \square A \rightarrow \square \diamond \square A$

$\left(\mathrm{T}^{*}\right): A / \square A$

9. $\square \diamond \square A \rightarrow \diamond \square A$

10. $\square \square \diamond \diamond \square A \rightarrow \diamond \square A$

11. $\square A \rightarrow \diamond \square \square A$

12. $\diamond \square A \rightarrow \diamond \diamond \square \square A$

13. $\square \square \diamond \diamond \square A \rightarrow \diamond \diamond \square \square A$

14. $\diamond \diamond \square(\diamond \square A \rightarrow \square A)$

15. $\square \diamond \diamond \square(\diamond \square A \rightarrow \square A)$

7 and the monotonicity rule $D^{*}: A / \square A$ the law of syllogism, 8 and 9 the equivalent version of $\left(\mathrm{T}^{*}\right)$

11 and monotonicity rule the law of syllogism, 10 and 12 (MP), 13 and 6 14 and Gödel's rule 
16. $\square \diamond \diamond \square(\diamond \square A \rightarrow \square A) \rightarrow \diamond \square(\diamond \square A \rightarrow \square A)$

17. $\diamond \square(\diamond \square A \rightarrow \square A)$

$\left(\mathrm{T}^{*}\right): A /(\square(\diamond \square A \rightarrow \square A))$

(MP), 15 and 16

It is easy to see that the rule $\left(\mathrm{RT}^{*}\right)$ is provable:

1. $\diamond \diamond A$

2. $\square \diamond \diamond A$

assumption

3. $\square \diamond \diamond A \rightarrow \diamond A$

Gödel's rule

4. $\diamond A$

(MP), 2 and 3

In the opposite direction we show that the formulas $\left(\mathrm{T}^{*}\right)$ and $\left(\mathrm{D}^{*}\right)$ belong to $\mathbf{S} \mathbf{5}_{\mathbf{M}}$. Indeed we have:

1. $\square(\square \diamond A \rightarrow \diamond A) \rightarrow \diamond(\square \diamond A \rightarrow \diamond A)$

(D): $A /(\square \diamond A \rightarrow \diamond A)$

2. $\diamond \square(\square \diamond A \rightarrow \diamond A) \rightarrow \diamond \diamond(\square \diamond A \rightarrow \diamond A)$

the monotonicity rule and 1

3. $\diamond \square(\square \diamond A \rightarrow \diamond A)$

$(\mathrm{MT}): A / \diamond A$

4. $\diamond \diamond(\square \diamond A \rightarrow \diamond A)$

(MP), 2 and 3

5. $\diamond(\square \diamond A \rightarrow \diamond A)$

$\left(\mathrm{RT}^{*}\right)$ and 4

6. $\diamond(\square \diamond A \rightarrow \diamond A) \rightarrow \diamond \diamond(\diamond A \rightarrow A)$

7. $\diamond \diamond(\diamond A \rightarrow A)$

otonicity rule

8. $\diamond(\diamond A \rightarrow A)$

(MP), 5 and 6

9. $\diamond(\diamond A \rightarrow A) \rightarrow(\square \diamond A \rightarrow \diamond A)$

$\left(\mathrm{RT}^{*}\right)$ and 7

10. $\square \diamond A \rightarrow \diamond A$

(MP), 8 and 9

In the proof of the schema $\left(\mathrm{T}^{*}\right)$ we use the schema $\left(\mathrm{D}^{*}\right)$.

1. $\square \diamond \diamond A \rightarrow \diamond \diamond A$

2. $\square \square \diamond \diamond A \rightarrow \square \diamond \diamond A$

3. $\square \square \diamond \diamond A \rightarrow \diamond \diamond A$

4. $(\square \square \diamond \diamond A \rightarrow \diamond \diamond A) \rightarrow \diamond(\square \diamond \diamond A \rightarrow \diamond A)$

5. $\diamond(\square \diamond \diamond A \rightarrow \diamond A) \rightarrow \diamond \diamond(\diamond \diamond A \rightarrow A)$

6. $\diamond \diamond(\diamond \diamond A \rightarrow A)$

7. $\diamond(\diamond \diamond A \rightarrow A)$

8. $\diamond(\diamond \diamond A \rightarrow A) \rightarrow(\square \diamond \diamond A \rightarrow \diamond A)$

9. $\square \diamond \diamond A \rightarrow \diamond A$
$\left(\mathrm{D}^{*}\right): A / \diamond A$

the monotonicity rule and 1 the law of syllogism, 2 and 1

$\mathbf{t} 5$

$\mathbf{t} 5$ and the monotonicity rule $2 \times(\mathrm{MP}), 3,4$ and 5 the rule $\left(\mathrm{RT}^{*}\right)$ and 6

(MP), 7 and 8

Proof of Theorem 12. Let $\diamond A \in \mathbf{S 5}$. There exists a S5-proof: $D_{1}, \ldots$, $D_{n}=\diamond A$. Let us consider a sequence of formulas $\diamond \square D_{1}, \ldots, \diamond \square D_{n}=$ $\diamond \square \diamond A$. We show the construction of the $\mathbf{S} \mathbf{5}_{\mathbf{M}}$-proof of the formula $\diamond \square \diamond A$. The proof goes by induction on $1 \leqslant i \leqslant n$. By the induction hypothesis, for each $k<i$ there exists in the sense of the logic $\mathbf{S} 5_{\mathbf{M}}$ a proof of the formula 
$\diamond \square D_{k}$. We show how to construct an analogous sequence for $\diamond \square D_{i}$. If in the given sequence the formula $D_{i}$ is either the axiom $(\mathrm{T})$ or $(5)$, then in both cases $\diamond \square D_{i}$ is also provable as an axiom of the logic $\mathbf{S} \mathbf{5}_{\mathbf{M}}$. If on the other hand $D_{i}$ is either the axiom $(\mathrm{K})$ or a classical theorem, then $\diamond \square D_{i}$ can be easily also proved in the sense of $\mathbf{S} 5_{\mathbf{M}}$ : we use Gödel's rule twice with $D_{i}$ obtaining in this way the antecedent of the substitution of the axiom (D): $\square \square D_{i} \rightarrow \diamond \square D_{i}$. The consequent of this substitution is the required formula. If, however, $D_{i}$ is of the form $\square D_{j}$, for some $j<i$, then by the induction hypothesis for $j$ there exists in $\mathbf{S} \mathbf{5}_{\mathbf{M}}$ a proof of formula $\diamond \square D_{j}$. By Lemma 8 and the monotonicity rule the given be the induction hypothesis sequence we can extend with steps $k+1-k+4$ :

k. $\quad \vdash_{\mathbf{S} 5_{\mathrm{M}}} \diamond \square D_{j}$

$\mathrm{k}+1 . \square D_{n} \rightarrow \diamond \square \square D_{j}$

$\mathrm{k}+2 . \diamond \square D_{j} \rightarrow \diamond \diamond \square \square D_{j}$

$\mathrm{k}+3 . \square \diamond \diamond \square \square D_{j}$

$\mathrm{k}+4 . \diamond \square \square D_{j}$ by the induction hypothesis the version of $T^{*}: A /\left(D_{j}\right)$ - Lemma 8 $\mathrm{k}+1$ and the monotonicity rule (MP), $\mathrm{k}, \mathrm{k}+2$ and $(\mathrm{RG})$ $\left(\mathrm{T}^{*}\right): A /\left(\square \square D_{j}\right),(\mathrm{MP})$ and $\mathrm{k}+3$

Let us consider the case that in the initial proof $D_{i}$ arises by the rule (MP), i.e., $\exists_{m, j<i} D_{m}=D_{j} \rightarrow D_{i}$, therefore by the induction hypothesis we have $\vdash_{\mathbf{S}_{\mathbf{M}}} \diamond \square D_{j}$ and $\vdash_{\mathbf{S} \mathbf{5}_{\mathbf{M}}} \diamond \square\left(D_{j} \rightarrow D_{i}\right)$. We associate the appropriate sequences, adding the following steps:

1. $\vdash_{\mathbf{S} 5_{\mathbf{M}}} \diamond \square D_{j} \quad$ by the induction hypothesis l+k. $\quad \leftarrow_{\mathbf{S} 5_{\mathrm{M}}} \diamond \square\left(D_{j} \rightarrow D_{i}\right) \quad$ by the induction hypothesis $\mathrm{l}+\mathrm{k}+1 . \quad \square\left(D_{j} \rightarrow D_{i}\right) \rightarrow \diamond \square \square\left(D_{j} \rightarrow D_{i}\right)$

the version of $\left(\mathrm{T}^{*}\right): A /\left(D_{j} \rightarrow D_{i}\right)$ $1+\mathrm{k}+2 . \quad \diamond \square\left(D_{j} \rightarrow D_{i}\right) \rightarrow \diamond \diamond \square \square\left(D_{j} \rightarrow D_{i}\right)$

$1+\mathrm{k}+3 . \quad \diamond \diamond \square \square\left(D_{j} \rightarrow D_{i}\right)$

$\mathrm{l}+\mathrm{k}+4 . \quad \square \square \diamond \square D_{j} \rightarrow \diamond \diamond \diamond \square D_{i}$

$1+\mathrm{k}+5 . \quad \square \square \diamond \square D_{j}$

$\mathrm{l}+\mathrm{k}+6 . \quad \diamond \diamond \diamond \square D_{i}$

$1+\mathrm{k}+7 . \quad \square \diamond \diamond \diamond \square D_{i}$

$1+\mathrm{k}+8 . \quad \square \diamond \diamond \diamond \square D_{i} \rightarrow \diamond \diamond \square D_{i}$

$\mathrm{l}+\mathrm{k}+9 . \quad \diamond \diamond \square D_{i}$

$1+\mathrm{k}+10 . \square \diamond \diamond \square D_{i}$

$1+\mathrm{k}+11 . \square \diamond \diamond \square D_{i} \rightarrow \diamond \square D_{i}$

$1+\mathrm{k}+12 . \diamond \square D_{i}$ $\mathrm{l}+\mathrm{k}+1$ and the monotonicity rule

(MP), $\mathrm{l}+\mathrm{k}$ and $\mathrm{l}+\mathrm{k}+2$

the axiom $(\mathrm{K})$ and $\mathbf{t} 1, \mathbf{t} 5$

$2 \times(\mathrm{RG})$ and $\mathrm{l}$.

(MP) and $\mathrm{l}+\mathrm{k}+4, \mathrm{l}+\mathrm{k}+5$

$(\mathrm{RG})$ and $\mathrm{l}+\mathrm{k}+6$

the axiom $\left(\mathrm{T}^{*}\right): A /\left(\diamond \square D_{i}\right)$

(MP), $\mathrm{l}+\mathrm{k}+7$ and $\mathrm{l}+\mathrm{k}+8$

(RG) and $\mathrm{l}+\mathrm{k}+9$

the axiom $\left(\mathrm{T}^{*}\right): A /\left(\square D_{i}\right)$

(MP), $\mathrm{l}+\mathrm{k}+10$ and $\mathrm{l}+\mathrm{k}+11$ 
which ends the inductive proof. So, for $i=n$ we have $\vdash_{\mathbf{S} 5_{\mathbf{M}}} \diamond \square \diamond A$. By the axiom $\left(\mathrm{D}^{*}\right)$, the provable in $\mathbf{S 5}_{\mathbf{M}}$ rule of monotonicity and (MP) we have: $\vdash_{\mathbf{S} 5_{\mathbf{M}}} \diamond \diamond A$, while by Gödel's rule, with the help of the axiom $\left(\mathrm{T}^{*}\right)$ we conclude that $\vdash_{\mathbf{S} 5_{\mathbf{M}}} \diamond A$. Since all our axioms belongs to the $M$-fragment of $\mathbf{S 5}$, so the postulated minimality $\mathbf{S} \mathbf{5}_{\mathbf{M}}$ is stated.

\subsection{Semantics of the logic $S 5_{M}$}

Now we give conditions for frames which establish the completeness result for the logic under consideration. We'll use Theorem 13 and the following observations semantically characterizing logic $\mathbf{K D}^{*} \mathbf{T}^{*}$.

Theorem 14. A formula is valid in all frames satisfying the condition

$$
\forall_{w} \exists \bar{w}\left(w R \bar{w} \wedge \forall_{w^{\prime}}\left(\bar{w} R w^{\prime} \rightarrow w R w^{\prime}\right)\right)
$$

iff it is provable in the logic $\mathbf{K}$ extended with $\left(\mathrm{D}^{*}\right)$, i.e., in $\mathbf{K}\left[\mathrm{D}^{*}\right]\left(=\mathbf{D}^{*}\right)$.

Proof. " $\Leftarrow$ " Using the standard procedure, via Lemma 34 from p. 26 it is enough to show that the axiom $\left(D^{*}\right)$ is valid in each frame satisfying the given condition. We assume to the contrary, that there is a frame satisfying the condition $(*)$, where the formula $\left(\mathrm{D}^{*}\right)$ is not valid, so there is a world $w$ and some valuation $v$, that $w \nvdash_{v}\left(\mathrm{D}^{*}\right)$, i.e., $w \models_{v} \square \diamond p$ and $w \nvdash_{v} \diamond p$. By the definition of the notion of the truth at a world for ' $\diamond$ ' we have $\bar{w} \not \nvdash_{v} p$ for all worlds $\bar{w}$, where $w R \bar{w}$; in particular, we have $w^{\prime} \nvdash_{v} p$ for the world $w^{\prime}$ postulated in the condition (*). By the assumption and the definition of truth for ' $\square$ ', we see that $w^{\prime} \models_{v} \diamond p$, thus there is $w^{\prime \prime}$, that $w^{\prime} R w^{\prime \prime}$ and $w^{\prime \prime} \models_{v}$ $p$, however by (*) we obtain $w R w^{\prime \prime}$, i.e., $w \models_{v} \diamond p$ which is a contradiction.

" $\Rightarrow$ " Let us consider the canonical model of the logic $\mathbf{D}^{*}$.

Firstly we show that for each world $w$, the set $\{A: \square A \in w\} \cup\{\square A$ : $\square A \in w\}$ is consistent with respect to $\mathbf{D}^{*}$. Assume otherwise, i.e., there are formulas $\square A_{1}, \ldots, \square A_{n}, \square A_{1}^{\prime}, \ldots, \square A_{m}^{\prime}, \in w$ that $\vdash_{\mathbf{D}^{*}} \neg\left(A_{1} \wedge \cdots \wedge A_{n} \wedge \square A_{1}^{\prime} \wedge\right.$ $\left.\cdots \wedge \square A_{m}^{\prime}\right)$. By classical logic we have $\vdash_{\mathbf{D}^{*}} A_{1} \wedge \cdots \wedge A_{n} \wedge A_{1}^{\prime} \wedge \cdots \wedge A_{m}^{\prime} \rightarrow$ $A_{1} \wedge \cdots \wedge A_{n}$, and on the basis of the logic $\mathbf{K}$ using $\mathbf{t} 2$ and obvious induction we get $\vdash_{\mathbf{D}^{*}} \square\left(A_{1} \wedge \cdots \wedge A_{n} \wedge A_{1}^{\prime} \wedge \cdots \wedge A_{m}^{\prime}\right) \rightarrow\left(\square A_{1} \wedge \cdots \wedge \square A_{n} \wedge \square A_{1}^{\prime} \wedge \cdots \wedge \square A_{m}^{\prime}\right)$, while using the absorption law for conjunction we obtain $\vdash_{\mathbf{D}^{*}} \square\left(A_{1} \wedge \cdots \wedge A_{n} \wedge\right.$ $\left.A_{1}^{\prime} \wedge \cdots \wedge A_{m}^{\prime}\right) \rightarrow\left(\square A_{1}^{\prime} \wedge \cdots \wedge \square A_{m}^{\prime}\right)$. Let us denote $A_{1} \wedge \cdots \wedge A_{n} \wedge A_{1}^{\prime} \wedge \cdots \wedge A_{m}^{\prime}$ as $A$. Our observations can be written as follows: $\vdash_{\mathbf{D}^{*}} A \rightarrow A_{1} \wedge \cdots \wedge A_{n}$ and $\vdash_{\mathbf{D}^{*}} \square A \rightarrow\left(\square A_{1}^{\prime} \wedge \cdots \wedge \square A_{m}^{\prime}\right)$.

Apparently $\square A \in w$; by the law of adjunction of implications we have: $\vdash_{\mathbf{D}^{*}} A \wedge \square A \rightarrow A_{1} \wedge \cdots \wedge A_{n} \wedge\left(\square A_{1}^{\prime} \wedge \cdots \wedge \square A_{m}^{\prime}\right)$, and via the law of 
contraposition $\vdash_{\mathbf{D}^{*}} \neg\left[A_{1} \wedge \cdots \wedge A_{n} \wedge\left(\square A_{1}^{\prime} \wedge \cdots \wedge \square A_{m}^{\prime}\right)\right] \rightarrow \neg(A \wedge \square A)$. Therefore, by the assumption and Modus Ponens, we have: $\vdash_{\mathbf{D}^{*}} \neg(A \wedge \square A)$, equivalently $\vdash_{\mathbf{D}^{*}} \square A \rightarrow \neg A$. By Gödel's rule and $\mathbf{t} 1$ we conclude $\vdash_{\mathbf{D}^{*}}$ $\diamond \square A \rightarrow \neg \square A$. Using Lemma 9 and the law of syllogism we get $\vdash \mathbf{D}^{*} \square A \rightarrow$ $\neg \square A$, i.e., $\neg \square A \in w$, therefore $\square A \notin w$. However $\square A \in w$, which is a contradiction.

We are ready to prove that the accessibility relation $R$ of the canonical model fulfills the condition (*). Since the set $\mathcal{W}=\{A: \square A \in w\} \cup\{\square A$ : $\square A \in w\}$ is consistent with respect to $\mathbf{D}^{*}$, there is thus a maximally consistent set $w^{\prime}$ containing $\mathcal{W}$, by the definition of the accessibility relation in the canonical model we state that $w R w^{\prime}$. Indeed, if $\square A \in w$, then $A \in \mathcal{W} \subseteq w^{\prime}$. Let $w^{\prime \prime}$ be any set that $w^{\prime} R w^{\prime \prime}$. We prove that $w R w^{\prime \prime}$. Let us assume that $\square B \in w$. By the definition of $w^{\prime}$ clearly $\square B \in w^{\prime}$, but since $w^{\prime} R w^{\prime \prime}$, so $B \in w^{\prime \prime}$.

Thus the canonical model of the logic $\mathbf{D}^{*}$ belongs to the class of models fulfilling $(*)$, then if some formula is valid in all frames satisfying the condition (*), it is also valid in the canonical model, but by Lemma 36 each formula valid in the canonical model, is provable in $\mathbf{D}^{*}$.

Theorem 15. A formula is valid in all frames fulfilling the condition

$$
\forall_{w} \exists \bar{w}\left(w R \bar{w} \wedge \forall_{w^{\prime}} \forall_{w^{\prime \prime}}\left(\bar{w} R w^{\prime} \wedge w^{\prime} R w^{\prime \prime} \rightarrow w R w^{\prime \prime}\right)\right)
$$

iff it is provable in the logic $\mathbf{K}$ with the axiom $T^{*}$, i.e., in $\mathbf{K}\left[\mathbf{T}^{*}\right]\left(=\mathbf{T}^{*}\right)$.

Proof. " $\Leftarrow$ " We prove the the axiom $T^{*}$ is valid in each frame fulfilling the given condition.

Assume to the contrary that there exists a frame fulfilling the condition $(\circledast)$, in which the formula $T^{*}$ is refuted. Then there is a world $w$ and valuation $v$, that $w \not \nvdash_{v} T^{*}$, i.e., $w \models_{v} \square \diamond \diamond p$ and $w \not \nvdash_{v} \diamond p$. The last condition via the definition of truth says that $\underline{w} \not \forall_{v} p$ for all possible worlds $\underline{w}$, such that $w R \underline{w}$, i.e., we also have $\bar{w} \nvdash_{v} p$ for $\bar{w}$, which is postulated by $(\circledast)$. By the assumption $w \models_{v} \square \diamond \diamond p$ and the conditions of truth for ' $\square$ ' for ' $\diamond$ ' there are worlds $w^{\prime}$ and $w^{\prime \prime}$, that $\bar{w} R w^{\prime}, w^{\prime} R w^{\prime \prime}$ and $w^{\prime \prime} \models_{v} p$, then by the condition $(\circledast)$ we get $w R w^{\prime \prime}$, i.e., $w \models_{v} \diamond p$ which is a contradiction.

" $\Rightarrow$ " We follow the proof of the previous theorem. Let us consider the canonical model of the logic $\mathbf{T}^{*}$. We prove the canonical frame satisfies $(\circledast)$. Let us start with the observation that for each possible world $w$, the set of formulas $\{A: \square A \in w\} \cup\{\square \square A: \square A \in w\}$ is $\mathbf{T}^{*}$-consistent. Assume otherwise, i.e., there are formulas $\square A_{1}, \ldots, \square A_{n}, \square A_{1}^{\prime}, \ldots, \square A_{m}^{\prime} \in w$ that 
$\vdash_{\mathbf{T}^{*}} \neg\left(A_{1} \wedge \cdots \wedge A_{n} \wedge \square \square A_{1}^{\prime} \wedge \cdots \wedge \square \square A_{m}^{\prime}\right)$. By the law of absorption we have $\vdash_{\mathbf{T}^{*}} A_{1} \wedge \cdots \wedge A_{n} \wedge A_{1}^{\prime} \wedge \cdots \wedge A_{m}^{\prime} \rightarrow A_{1} \wedge \cdots \wedge A_{n}$, while on the basis of $\mathbf{K}$, and via $\mathbf{t} 2$ we get $\square \square\left(A_{1} \wedge \cdots \wedge A_{n} \wedge A_{1}^{\prime} \wedge \cdots \wedge A_{m}^{\prime}\right) \rightarrow$ $\left(\square \square A_{1} \wedge \cdots \wedge \square \square A_{n} \wedge \square \square A_{1}^{\prime} \wedge \cdots \wedge \square \square A_{m}^{\prime}\right)$, thus once more using the law of absorption for conjunction we see that $\vdash_{\mathbf{K}} \square \square\left(A_{1} \wedge \cdots \wedge A_{n} \wedge A_{1}^{\prime} \wedge \cdots \wedge A_{m}^{\prime}\right) \rightarrow$ $\left(\square \square A_{1}^{\prime} \wedge \cdots \wedge \square \square A_{m}^{\prime}\right)$. If we denote the formula $A_{1} \wedge \cdots \wedge A_{n} \wedge A_{1}^{\prime} \wedge \cdots \wedge A_{m}^{\prime}$ by $A$, then the above observations can be written: $\vdash_{\mathbf{T}^{*}} A \rightarrow A_{1} \wedge \cdots \wedge$ $A_{n}$ and $\vdash_{\mathbf{T}^{*}} \square \square A \rightarrow\left(\square \square A_{1}^{\prime} \wedge \cdots \wedge \square \square A_{m}^{\prime}\right)$. Clearly $\square A \in w$, indeed since $\square A_{1}, \ldots, \square A_{n}, \square A_{1}^{\prime}, \ldots, \square A_{m}^{\prime} \in w$, thus via the law of adjunction also $\square A_{1} \wedge \cdots \wedge \square A_{n} \wedge \square A_{1}^{\prime} \wedge \cdots \wedge \square A_{m}^{\prime} \in w$. In $\mathbf{K}$ we have $\square A_{1} \wedge \cdots \wedge \square A_{n} \wedge$ $\square A_{1}^{\prime} \wedge \cdots \wedge \square A_{m}^{\prime} \rightarrow \square\left(A_{1} \wedge \cdots \wedge A_{n} \wedge A_{1}^{\prime} \wedge \cdots \wedge A_{m}^{\prime}\right)$, so $\square\left(A_{1} \wedge \cdots \wedge A_{n} \wedge\right.$ $\left.A_{1}^{\prime} \wedge \cdots \wedge A_{m}^{\prime}\right) \in w$; and using the law of addition of implications we state: $A \wedge \square \square A \rightarrow A_{1} \wedge \cdots \wedge A_{n} \wedge\left(\square \square A_{1}^{\prime} \wedge \cdots \wedge \square \square A_{m}^{\prime}\right)$, by the contraposition law and the assumption we have: $\vdash_{\mathbf{T}^{*}} \neg(A \wedge \square \square A)$, equivalently $\vdash_{\mathbf{T}^{*}} \square \square A \rightarrow \neg A$. By Gödel's rule and $\mathbf{t} 1$ we deduce that $\vdash_{\mathbf{T}^{*}} \diamond \square \square A \rightarrow \neg \square A$. By Lemma 8 we have $\vdash_{\mathbf{T}^{*}} \square A \rightarrow \diamond \square \square A$, and by the law of syllogism we get $\vdash_{\mathbf{T}^{*}} \square A \rightarrow \neg \square A$, i.e., $\neg \square A \in w$, contrary to the earlier observation.

We show that canonical frame of the logic $\mathbf{T}^{*}$ satisfies the condition $(\circledast)$. The set $\{A: \square A \in w\} \cup\{\square \square A: \square A \in w\}$ is consistent with respect to $\mathbf{T}^{*}$, so it is contained in a maximally consistent set. Let us denote it by $\bar{w}$. By the definition of the accessibility relation in the canonical frame we get $w R \bar{w}$. Let $w^{\prime}, w^{\prime \prime}$ be any possible world such that $\bar{w} R w^{\prime}$ and $w^{\prime} R w^{\prime \prime}$. We show that $w R w^{\prime \prime}$. Let us assume that $\square B \in w$, by the definition of the world $\bar{w}$ we see that $\square \square B \in \bar{w}$, by via the assumption about $w^{\prime}$ and $w^{\prime \prime}$ we get $\square B \in w^{\prime}$ and $B \in w^{\prime \prime}$, since $B$ was any formula, we have $w R w^{\prime \prime}$. The rest of the proof follows in the standard way, as in the previous theorem.

The above semantical conditions express a kind of weaker version of the condition of transitivity of the relation $R$. Therefore obviously these theorems can be generalized:

Theorem 16. A formula is valid in all frames fulfilling the condition

$$
\forall_{w} \exists \bar{w}\left(w R \bar{w} \wedge \forall_{w_{1}} \forall_{w_{2}}\left(\bar{w} R w_{1} \wedge w_{1} R w_{2} \wedge \ldots w_{n-1} R w_{n} \rightarrow w R w_{n}\right)\right)
$$

iff it is provable in logic $\mathbf{K}$ with the axiom $\square \underbrace{\otimes \ldots \diamond}_{n} A \rightarrow \diamond A$.

Proof. It is analogous.

There follows an easily-provable corollary: 
Corollary 17. Formulas $\left(\mathrm{T}^{*}\right)$ and $\left(\mathrm{D}^{*}\right)$ are independent on the basis of $\mathbf{K}$.

Proof. We point out a model whose frame fulfills (*) but does not fulfill the condition $(\circledast)$.

$W:=\left\{w, w_{1}, w_{2}, w_{3}\right\}$, the relation $R$ between possible worlds is indicated with arrows, which ends in the second argument of an ordered pair. One can easily see that in the world $w$ formula $\square \diamond \diamond p \rightarrow \diamond p$ is not satisfied, while formula $D^{*}$ is true in the model.

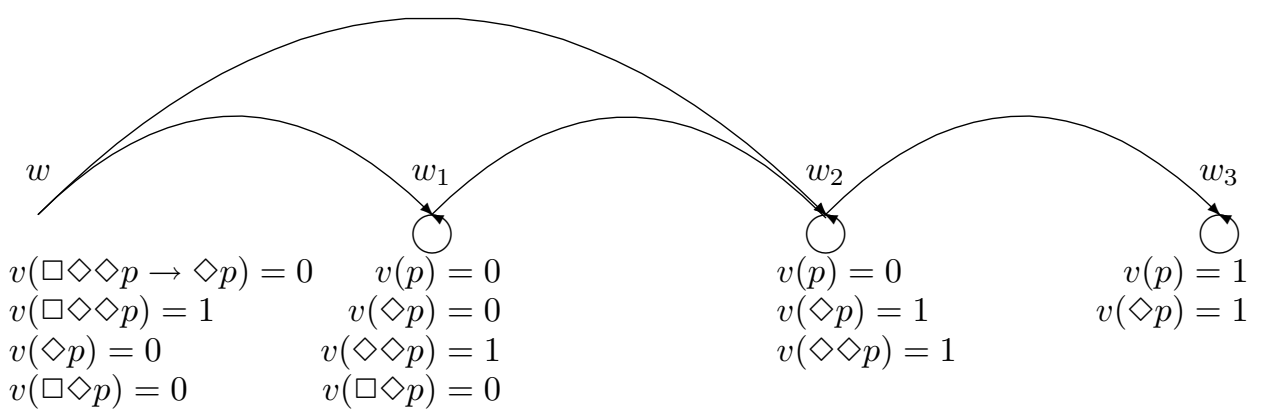

Now let us consider a model which for which the condition $(\circledast)$ is satisfied, and the condition $(*)$ is not satisfied. Let $W=\left\{w, w_{1}, w_{2}, w_{3}\right\}$. In each world the formula $\left(\mathrm{T}^{*}\right)$ is satisfied, while the formula $D^{*}$ is not satisfied in the world $w$.

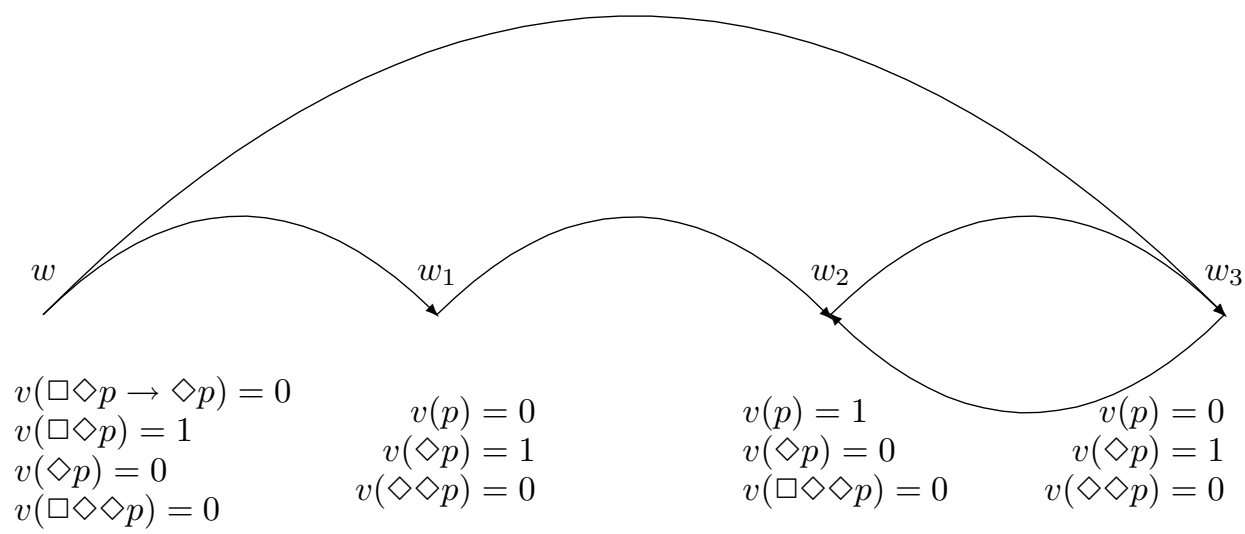




\section{Appendix: Preliminaries in modal logic}

\section{Syntax}

By a logic we mean any set of formulas, which is closed under the substitution rule and the consequence relation.

We recall standard notions concerning modal logics. The language of a modal logic arises by enriching the standard propositional language with the unary logical constant ' $\diamond$ ' (so called possibility) or ' $\square$ ' (necessity), which are inter-definable by the following condition:

$$
\diamond A \leftrightarrow \sim \square \sim A .
$$

We say that ' $\diamond$ ' and ' $\square$ ' are dual connectives.

By the logic $\mathbf{K}$ we mean the smallest modal logic containing the classical logic, the Kripke axiom

$$
\square(A \rightarrow B) \rightarrow(\square A \rightarrow \square B),
$$

closed under rules of Modus Ponens (MP) and the Gödel's rule:

$$
\frac{A}{\square A}
$$

A normal modal logic is a set of formulas which contains $\mathbf{K}$ and is closed under the same as $\mathbf{K}$ rules.

By the logic $\mathbf{D}$ we mean the minimal normal logic which contains the axiom $^{3}$ :

$$
\square A \rightarrow \diamond A
$$

The logic $\mathbf{T}$ is the normal modal logic obtained by adding to $\mathbf{K}$, the axiom:

$$
\square A \rightarrow A \text { or equivalently } A \rightarrow \diamond A
$$

The logic $\mathbf{S} 4$ is the normal modal logic defined by adding to $\mathbf{T}$, the axiom

$$
\diamond \diamond A \rightarrow \diamond A \text { or equivalently } \square A \rightarrow \square \square A .
$$

The logic $\mathbf{S} 5$ is the normal modal logic defined by adding to $\mathbf{T}$, the axiom

$$
\diamond \square A \rightarrow \square A \text { or equivalently } \diamond A \rightarrow \square \diamond A \text {. }
$$

\footnotetext{
${ }^{3}$ We say that this normal logic is defined by adding to $\mathbf{K}$ the axiom $\left(D^{*}\right)$.
} 
The logic Triv is the normal modal logic defined by adding to $\mathbf{D}$, the axiom

$$
A \rightarrow \square A \text { or equivalently } \diamond A \rightarrow A .
$$

For each of those logics one can define a consequence relation allowing (MP) to be the only rule of inference. For example we have:

Definition 18. We say that a given formula $A$ is $\mathbf{S 5}$-provable on the basis of $X$ (notation: $X \vdash_{\mathbf{S 5}} A$ ) iff there is a sequence of formulas $C_{1}, \ldots, C_{n}=A$, where for each $1 \leqslant i \leqslant n$ : $C_{i} \in X$ either $C_{i}$ is a theorem of $\mathbf{S 5}$, or arises by (MP) from earlier formulas in the that sequence, ${ }^{4}$

Let us recall some well-known theorems and rules provable in normal modal logics which will be needed in the sequel.

Lemma 19. The following rule are provable in all normal logics

1. The monotonicity rule: $\frac{A \rightarrow B}{\square A \rightarrow \square B}$,

2. The dual form of the monotonicity rule: $: \frac{A \rightarrow B}{\diamond A \rightarrow \diamond B}$,

3. The extensionality: $\frac{A \leftrightarrow B}{\square A \leftrightarrow \square B}$,

4. The dual form of extensionality: $\frac{A \leftrightarrow B}{\diamond A \leftrightarrow \diamond B}$.

LEMMA 20. The following formulas are theorems in all normal logics

$$
\square(A \rightarrow B) \rightarrow(\diamond A \rightarrow \diamond B),
$$

(t3) The dual form of the regularity: $\diamond(A \vee B) \leftrightarrow(\diamond A \vee \diamond B)$

$(\mathbf{t} 4) \quad \square(A \vee B) \rightarrow(\square A \vee \diamond B), \square(A \vee B) \rightarrow(\diamond A \vee \square B)$,

$(\mathbf{t} 5) \quad \diamond(A \rightarrow B) \leftrightarrow(\square A \rightarrow \diamond B)$,

$(\mathbf{t} 6) \quad \diamond(A \wedge B) \rightarrow(\diamond A \wedge \diamond B)$,

(t7) $\quad \diamond A \wedge \square B \rightarrow \diamond(A \wedge B)$,

$$
\square A \leftrightarrow \neg \diamond \neg A
$$

$\perp$ can be defined as the abbreviation of: $p \wedge \sim p$ (or any other contrtautology).

\footnotetext{
${ }^{4}$ Notice that we do not use Gödel's rule here.
} 
LEMma $21 . \vdash_{\mathbf{K}} \diamond \perp \leftrightarrow \perp$

ProOF.

1. $\perp \rightarrow \diamond \perp$

2. $\sim \perp$

3. $\square \sim \perp$

4. $\sim \perp \rightarrow \square \sim \perp$

5. $\sim \square \sim \perp \rightarrow \sim \sim \perp$

6. $\diamond \perp \rightarrow \perp$ by the classical logic $\perp \rightarrow A$ by the classical logic 2 and $(\mathrm{RG})$

3 and weakening

4 and the law of contraposition $5,(1)$ and the law of double negation

LEMMA 22. $\vdash_{\text {S5 }} \diamond \diamond A \rightarrow \diamond A$, i.e., $(S 5) \vdash(4)$.

Proof.

1. $\diamond A \rightarrow \square \diamond A$

the equivalent version of 5

2. $\diamond \diamond A \rightarrow \diamond \square \diamond A$

Lemma 2

3. $\diamond \square \diamond A \rightarrow \square \diamond A$

4. $\square \diamond A \rightarrow \diamond A$

the substitution of the axiom (5): $A / \diamond A$

5. $\diamond \diamond A \rightarrow \diamond A$

the instance of $(\mathrm{T}): A / \diamond A$ the law of syllogism and 2, 3, 4

LEMMA 23. $\vdash_{\mathrm{S} 5} \diamond(\diamond A \rightarrow B) \leftrightarrow(\diamond A \rightarrow \diamond B)$

Proof. follows from two above theorems: $\vdash_{\mathbf{K}} \diamond(\diamond A \rightarrow B) \leftrightarrow(\square \diamond A \rightarrow$ $\diamond B)$ and $\vdash_{\mathbf{S 5}}(\square \diamond A \rightarrow \diamond B) \leftrightarrow(\diamond A \rightarrow \diamond B)$.

LEMMA 24. $\vdash_{\mathbf{S 5}} \diamond(A \wedge \diamond B) \leftrightarrow(\diamond A \wedge \diamond B)$.

Proof. " $\Rightarrow$ " By t6 we have $\vdash_{\mathbf{K}} \diamond(A \wedge \diamond B) \rightarrow \diamond A \wedge \diamond \diamond B$. And by Lemma 22 we obtain $\vdash_{\text {S5 }} \diamond A \wedge \diamond \diamond B \rightarrow \diamond A \wedge \diamond B$.

" $\Leftarrow$ " Using classical logic and $S 5$ we have $\vdash_{\mathrm{S} 5}(\diamond A \wedge \diamond B) \rightarrow(\diamond A \wedge \square \diamond B)$, and substituting $\diamond B$ for $B$ in the formula $\mathbf{t} 7$ we see that $\vdash_{\mathbf{K}} \diamond A \wedge \square \diamond B \rightarrow$ $\diamond(A \wedge \diamond B)$.

Let us introduce the following notation:

For any modal logic $\boldsymbol{P}$, we define the set $\diamond \boldsymbol{P}:=\{\diamond A: \diamond A \in \boldsymbol{P}\}$ which is called the $M$-fragment of the logic $\boldsymbol{P}$.

For any modal logic $\boldsymbol{P}$, let $\mathrm{M}(\mathbf{P}):=\left\{A \in\right.$ For $\left._{\mathrm{m}}: \diamond A \in \boldsymbol{P}\right\}$, where For $_{\mathrm{m}}$ is the set of all modal formulas. The set $\mathrm{M}(\boldsymbol{P})$ is called M-analogon of the $\operatorname{logic} \boldsymbol{P}$. 


\section{Semantics}

In this section we present the Kripke semantics for modal logics. Once more we start with some standard definitions, which will be used later.

DeFinition 25. A relational frame (in shortage a frame) is a pair $\langle W, R\rangle$ consisting of nonempty set $W$, and a binary relation $R$ on $W$. Elements of the set $W$ we call the points of the frame, while $R$ is the accessibility relation.

Definition 26. A valuation is any function $v$ : Var $\longrightarrow 2^{W}$.

Definition 27. A model is a triple $\langle W, R, v\rangle$, where $\langle W, R\rangle$ is a frame and $v$ is a valuation. We say that $\langle W, R, v\rangle$ is based on the frame $\langle W, R\rangle$.

Definition 28. A formula $A$ is true in the point $w \in W$ under the valuation $v$ (notation: $w \models_{v} A$ ) iff

1. if $A$ is a propositional letter $p$, then:

$w \models{ }_{v} p \Leftrightarrow w \in v(p)$.

2. if $A$ is of the form $\neg B$, for some formula $B$, then:

$w \models_{v} \neg B \Leftrightarrow$ it is not the case that $w \models_{v} B$ (as abbreviation we use $\left.w \not \vee_{v} B\right)$.

3. if $A$ is of the form $B \wedge C$, for some formulas $B$ and $C$, then $w \models_{v} B \wedge C \Leftrightarrow w \models_{v} B$ and $w \models_{v} C$.

4. if $A$ is of the form $B \vee C$, for some formulas $B$ and $C$, then $w \models{ }_{v} B \vee C \Leftrightarrow w \models{ }_{v} B$ or $w \models{ }_{v} C$.

5. if $A$ is of the form $B \rightarrow C$, for some formulas $B$ and $C$, then $w \models_{v} B \rightarrow C \Leftrightarrow w \not{ }_{v} B$ or $w \models_{v} C$.

6. if $A$ is of the form $B \leftrightarrow C$, for some formulas $B$ and $C$, then $w \models_{v} B \leftrightarrow C \Leftrightarrow\left(w \models_{v} B\right.$ and $\left.w \models_{v} C\right)$ or $\left(w \not \models_{v} B\right.$ and $\left.w \not \models_{v} C\right)$.

7. if $A$ is of the form $\diamond B$, for a formula $B$, then $w \models{ }_{v} \diamond B \Leftrightarrow \exists_{w^{\prime} \in W}\left(w R w^{\prime} \wedge w^{\prime} \models_{v} B\right)$.

8. if $A$ is of the form $\square B$, for a formula $B$, then $w \models_{v} \square B \Leftrightarrow \forall_{w^{\prime} \in W}\left(w R w^{\prime} \Rightarrow w^{\prime} \models_{v} B\right)$. 
Definition 29. A formula $A$ is true in a model $M=\langle W, R, v\rangle$ (notation $M \models A$ ) iff $w \models{ }_{v} A$ for each $w \in W$.

Definition 30. A formula $A$ is valid in frame $\langle W, R\rangle$ iff it is true in all models based on $\langle W, R\rangle$.

Definition 31. 1. Logic $\boldsymbol{P}$ is complete with respect to the class of frames $\mathcal{C}$ iff (a formula $A$ is a theorem of $\boldsymbol{P}$ iff $A$ is valid in each frame from $\mathcal{C}$. In that case we say that frames of the class $\mathcal{C}$ are $\boldsymbol{P}$-frames.

2. Any frame in which all theorems of a given modal logic $\boldsymbol{P}$ are valid we call a frame for $\boldsymbol{P}$.

Definition 32. $A$ is a S5-consequence of the set $X$ (notation: $X \models_{\mathbf{S 5}} A$ ) iff for any model $\langle W, R, v\rangle$ with equivalence accessibility-relation and for any $w \in W$ if $w \models_{v} X$ then $w \models_{v} A$.

Lemma 33. 1. S5-frames are frames with the equivalence accessabilityrelation.

2. $X \vdash_{\mathbf{S} 5} A$ iff $X \models_{\mathbf{S} 5} A$.

Let us recall the classical:

Lemma 34. (a) The axiom $K$ is valid in any frame.

(b) All classical tautologies are valid in each frame.

(c) The set of all formulas valid in a given frame is closed under Gödel's rule, (MP) and substitution.

Definition 35. The set $X$ of formulas is inconsistent with respect to the modal logic $\boldsymbol{P}$ (or shortly $\boldsymbol{P}$-inconsistent) iff there are formulas $A_{1}, \ldots A_{n} \in$ $X$, such that $\vdash_{\boldsymbol{P}} \neg\left(A_{1} \wedge \cdots \wedge A_{n}\right)$. The set $X$ is consistent with respect to the modal logic $\boldsymbol{P}$ (or shortly $\boldsymbol{P}$-consistent) iff it not $\boldsymbol{P}$-inconsistent.

We have also:

Lemma 36. All maximally consistent sets with respect to the modal logic $\boldsymbol{P}$ contain $\boldsymbol{P}$ and are closed under (MP).

Finally let us recall the definition of a compact logic:

Definition 37. A logic $\boldsymbol{P}$ is compact iff for every $\boldsymbol{P}$-consistent set of formulas $X$, there is a world $w$ in some model $M$ based on a frame for $\boldsymbol{P}$, such that all the formulas in $X$ are true in that world in $M$. 


\section{Canonical models}

Definition 38. Let $\boldsymbol{P}$ be any modal logic.

1. Let $W$ be a class of all maximally consistent sets with respect to $\boldsymbol{P}$, and $R$ be a binary relation on $W$ defined as follows: $w R w^{\prime} \Leftrightarrow \forall_{A}(\square A \in w \Rightarrow$ $A \in w)$. The canonical frame of the logic $\boldsymbol{P}$ is the pair $\langle W, R\rangle$.

2. A canonical model of the logic $\boldsymbol{P}$ is the model $\langle W, R, v\rangle$, where $\langle W, R\rangle$ is the canonical frame of the logic $\boldsymbol{P}$ and the following condition is satisfied for any variable $p_{i}$ :

$$
v\left(p_{i}\right)=\left\{w \in W: p_{i} \in w\right\} .
$$

i.e., $w \models_{v} p_{i} \Leftrightarrow p_{i} \in w$.

We have:

Lemma 39. Let $\langle W, R, v\rangle$ be a canonical model of the logic $\boldsymbol{P}$.

(a) For each formula $A$ and $w \in W$ the following holds: $w \models_{v} A \Leftrightarrow A \in w$.

(b) A formula is true in $\langle W, R, v\rangle$ iff it is a theorem of $\boldsymbol{P}$.

Let us stress that if the notion of truth in the canonical frame instead of truth in the canonical model were used, the point (b) of the above theorem would not hold in general. This is why the important notion of canonical logic is often formulated:

Definition 40. A logic $\boldsymbol{P}$ is canonical iff all theorems of $\boldsymbol{P}$ are valid in the canonical frame of $\boldsymbol{P}$, i.e., if the canonical frame of $\boldsymbol{P}$ is a frame for $\boldsymbol{P}$.

Acknowledgments. The research for this paper was indirectly financed by the Flemish Minister responsible for Science and Technology (contract BIL $98 / 37)$.

\section{References}

[1] Batens, D., "Dialectical dynamics within formal logics", Logic et Analyse 114, (1986), 161-173.

[2] Batens, D., "Dynamic dialectical logics", in G. Priest, R. Routley and J. Norman (eds.), Paraconsistent Logic. Essays on the Inconsistent, Philosophia Verlag, München, pp. 187-217. 
[3] Batens, D., "Inconcistency adaptive logics", in E. Orłowska (ed.), Logic at Work. Essays Dedicated to the Memory of Helena Rasiowa, Physica Verlag (Springer), Heidelberg - New York, 1999, pp. 445-472.

[4] Błaszczuk, J. J., W. Dziobiak: "Modal logics connected with systems $\mathbf{S} \mathbf{4}_{n}$ of Sobociński", Bulletin of the Section of Logic 4, 3 (1975), 103-108.

[5] Chagrov, A., and M. Zakharyaschev, Modal Logic, Oxford Science Publications, Clarendon Press, Oxford, 1997.

[6] Fine, K., "Normal forms in modal logic", Notre Dame Journal of Formal Logic 16 (1975), 229-237.

[7] Furmanowski, T., Remarks on Discussive Propositional Calculus, Routledge, New Your, London 1996.

[8] Goldblatt, R., "First-order definability in modal logic", The Journal of Symbolic Logic 40 (1975), 35-40.

[9] Goldblatt, R., "The KcKinsey Axiom is not canonical", The Journal of Symbolic Logic 56 (1991), 554-562.

[10] Hughes, G. E., and M. J. Cresswell: A New Introduction to Modal Logic, Routledge, New Your, London 1996.

[11] Jaśkowski, S., "Rachunek zdań dla systemów dedukcyjnych sprzecznych", Studia Societatis Scientiarum Torunensis, Sect. A, I, No 5 (1948), 57-77. In english: "A propositional calculus for inconsistent deductive systems", Logic and Logical Philosophy 7 (1999), 35-56.

[12] Jaśkowski, S., "O koniunkcji dyskusyjnej w rachunku zdań dla systemów dedukcyjnych sprzecznych", Studia Societatis Scientiarum Torunensis, Sect. A, I, No 8 (1949), 171-172. In english: "On the discussive conjunction in the propositional calculus for inconsistent deductive systems", Logic and Logical Philosophy 7 (1999), 57-59.

[13] Lemmon, F. J., An Introduction to Modal Logic, American Philosophical Quarterly Monograph Series, vol. 11, Basil Balckwell, Qxford 1977.

[14] Perzanowski, J., "On M-fragments and L-fragments of normal modal propositional logics", Reports on Mathematical Logic 5 (1975), 63-72.

[15] Wang, X.,: "The KcKinsey Axiom is not compact", The Journal of Symbolic Logic 57 (1992), 1230-1238.

Marek Nasieniewski

Department of Logic

N. Copernicus University

ul. Asnyka $2 \mathrm{~b}$

87-100 Toruń, Poland

mnasiem@cc.uni.torun.pl 